\title{
Adaptação ao Divórcio e Relações Coparentais: Contributos da Teoria da Vinculação
}

\author{
Adjustment to Divorce and Co-parental Relations: \\ Contributions from the Theory of Attachment
}

\author{
Diogo Lamela ${ }^{*}, a, b$, Bárbara Figueiredo ${ }^{a}, \&$ Alice Bastos $^{b}$ \\ ${ }^{a}$ Universidade do Minho, Braga, Portugal \& ${ }^{b}$ Instituto Politécnico de Viana do Castelo, Viana do Castelo, Portugal
}

\begin{abstract}
Resumo
Neste artigo propõe-se uma contribuição da teoria da vinculação na compreensão dos processos de adaptação dos adultos ao seu divórcio e como a desvinculação ao ex-cônjuge interfere na coparentalidade pós-divórcio. Este artigo formula duas hipóteses teóricas. A primeira hipótese afirma que o divórcio, enquanto processo relacional, deve ser lido como um momento de perda que germina reacções psicológicas similares às experienciadas pelos viúvos, tal como descreve Bowlby no modelo de perda da figura de vinculação, estando dependente dos estilos de vinculação dos adultos. A segunda hipótese sustenta que a coparentalidade pós-divórcio é predita pelos estilos de vinculação e pela qualidade da reorganização da vinculação dos pais. Finalmente, uma integração teórica é apresentada, operacionalizada numa proposta de investigação futura neste domínio.

Palavras-chave: Divórcio; Vinculação; Psicopatologia; Adaptação; Coparentalidade.
\end{abstract}

\begin{abstract}
In this article, it is proposed the contribution of the attachment theory for understanding adults' adjustment processes to their divorce and how detachment to ex-spouse can infer in co-parenting relationships after marital dissolution. This article makes two theoretical assumptions that focus on two dimensions. The first hypothesis states that the divorce, while a relational process, should be read as a moment of loss that germinates similar psychological reactions to those experienced by widows. Bowlby describes it in his model of loss of the attachment figure as dependent on attachment styles of divorced adults. The second hypothesis argues that the post-divorce co-parenting relationships are predicted by the attachment styles and by the quality of parents' attachment reorganization. At the end, a theoretical integration is built, based on a proposal for future research in this area.

Keywords: Divorce; Attachment; Psychopathology; Adjustment; Co-parenting.
\end{abstract}

Holmes e Rahe (1967), no seu estudo de referência, mostraram que o divórcio é o segundo stressor mais destruturante na vida adulta, imediatamente a seguir à morte do cônjuge. Na perspectiva da teoria da vinculação, não é surpreendente que, na longa lista de potenciais estressores descritos empiricamente, duas situações de separação/perda encabecem os eventos de vida percepcionados pelos adultos como os mais exigentes do ponto de vista da sua reorganização psicossocial. A maioria dos estudos conclui que as pessoas divorciadas experienciam menor bem-estar psicológico, piores níveis de felicidade e maiores índices de sintomatologia psicológica, nomea-

\footnotetext{
${ }^{*}$ Endereço para correspondência: Universidade do Minho, Instituto de Educação e Psicologia, Serviço de Consulta Psicológica e Desenvolvimento Humano - Unidade de Crianças e Adolescentes, Campus Gualtar, Braga, Portugal, 4710-057.E-mail:dlamela@ese.ipvc.pt

Este estudo foi suportado por uma Bolsa de Doutoramento ao primeiro autor pela Fundação para a Ciência e Tecnologia de Portugal (SFRH/BD/43525/2008).
}

damente depressão e ansiedade, quando comparadas às pessoas que permanecem casadas. Os adultos divorciados reportam mais distress psicofisiológico, perdas acentuadas na segurança económica e financeira e no suporte social, alterações depreciativas na percepção do self e desestabilização emocional nos sistemas de procura e prestação de cuidados (Braver, Shapiro, \& Goodman, 2006; Gähler, 2006). No entanto, a intensidade, severidade e duração dos níveis de desajustamento ao divórcio estão dependentes, entre outros factores, de qual o membro da ex-díade iniciou o processo de divórcio e a duração do casamento (Kalmijn \& Poortman, 2006; Wang \& Amato, 2000).

Apesar de existirem dados empíricos sobre as respostas ao divórcio, pouco é sabido sobre os seus processos explicativos (Davis, Shaver, \& Vernon, 2003). Conceptualizando o divórcio como um processo relacional, o presente artigo teórico tem como finalidade contribuir para o enriquecimento deste domínio, explorando a hipótese principal que a qualidade da vinculação ao ex-cônjuge 
durante o casamento pode explicar e predizer os níveis de ajustamento após a dissolução do casamento. Apresentamos uma segunda hipótese que sustenta que as relações coparentais estarão dependentes do processo de desvinculação ao ex-cônjuge que, por sua vez, é resultante dos estilos de vinculação dos adultos. Assim, o divórcio apresenta-se como uma situação desenvolvimental apropriada para melhor compreender como as estratégias de vinculação podem relacionar-se com a adaptação psicológica à dissolução de relações interpessoais significativas, bem como na gestão das relações coparentais.

Mais especificamente, os objectivos deste artigo são dois: (a) clarificar e definir limites conceptuais dos construtos de divórcio, coparentalidade e vinculação e (b) elucidar sobre como o divórcio pode ser considerado como um evento na vida adulta que activa o sistema de vinculação e, dessa forma, condicionar e especificar a qualidade das relações coparentais após a dissolução conjugal. Estas hipóteses teóricas são discutidas a partir de duas diferentes perspectivas, no entanto relacionadas: (a) quais os contributos da vinculação para a compreensão da adaptação individual e familiar ao divórcio e (b) quais as direcções para a investigação futura da intersecção teórica entre a vinculação com o divórcio e a coparentalidade.

O presente artigo está organizado em quatro momentos. Num primeiro momento, são expostos os pressupostos essenciais do divórcio, da coparentalidade e da vinculação na vida adulta, construtos basilares das hipóteses teóricas que apresentaremos durante o artigo. Ainda no primeiro momento, apresentaremos as formulações da teoria da vinculação sobre os processos de (des)ajustamento psicológico e psicopatologia. Este tópico torna-se relevante, uma vez que irá permitir enquadrar a sintomatologia psicopatológica apresentada pela maioria dos divorciados à luz da vinculação e do processo de perda de Bowlby (1980). O segundo momento do artigo será dispendido na apresentação da nossa hipótese de a ciência psicológica conceptualizar o divórcio como um processo de (des)vinculação e como o processo de ajustamento à dissolução está baseada nos estilos de vinculação dos adultos e as respostas de ajustamento como correlato do processo de perda de uma figura de vinculação. Num terceiro momento, levantamos a hipótese que os estilos de vinculação e o processo de desvinculação dos pais funcionam como o principal preditor da qualidade das relações coparentais e do funcionamento familiar após o divórcio. Finalmente, no quarto momento, pretende-se integrar as hipóteses teóricas levantadas, propondo agendas de investigação neste estimulante e embrionário campo de investigação.

\section{Divórcio e Coparentalidade}

Para além das implicações jurídicas e sociais, o divórcio começa a ser validado pela Psicologia e Psicopatologia do Desenvolvimento como uma das principais transições não-normativas na vida adulta (Cavanaugh \& BlanchardFields, 2009). A literatura científica sobre divórcio sustenta que a dissolução conjugal é um exigente desafio para a regulação desenvolvimental, que obriga ao adulto a activação da maioria dos seus sistemas comportamentais e cognitivos para conter os seus efeitos negativos e potenciar uma adaptação saudável à nova condição familiar.

Por definição da ciência psicológica, o divórcio é um evento que pode funcionar como um marcador desenvolvimental para mudanças positivas e negativas nos percursos de vida dos adultos que o experienciam (Hetherington \& Kelly, 2002). Perspectivando o divórcio como marcador de mudanças de rotas desenvolvimentais, a investigação das últimas décadas tem evidenciado resultados aparentemente contraditórios - caso sejam lidos linearmente sobre os efeitos do divórcio nos adultos: se, por um lado, a dissolução conjugal pode produzir eventos agudos e/ou crónicos de desajustamento pessoal, como, por exemplo, diminuição acentuada dos recursos financeiros disponíveis, problemas de saúde, aumento da sintomatologia psicopatológica e diminuição das competências parentais (Braver et al., 2006), por outro lado, pode contribuir para maiores sentimentos de bem-estar e realização pessoal, construção de novas relações íntimas mais satisfatórias e funcionar como um factor de agência para o crescimento e complexidade pessoais (King \& Raspin, 2004; Lamela, 2009). Esta dualidade desenvolvimental entre efeitos positivos e/ou negativos não é aleatória, sendo que a pesquisa empírica tem demonstrado que tal dualidade é consequência da capacidade individual na utilização dos recursos pessoais (e.g., estilos interpessoais, estratégias de coping e competências de regulação emocional e comportamental) e contextuais (e.g., suporte social, serviços públicos e psicoterapia) durante e após o divórcio (Hetherington, 2006).

Por consequência, tendo como referência o adulto divorciado, e utilizando uma matriz desenvolvimental, esta diversidade nas respostas ao divórcio parece ser o resultado da plasticidade do adulto e do seu sistema desenvolvimental (Greene, Anderson, Hetherington, Forgatch, \& DeGarmo, 2006; Hetherington \& Kelly, 2002) em que as possibilidades de mudança positiva estão dependentes de múltiplas características individuais e contextuais. Tal como defenderemos ao longo deste artigo, devido à natureza relacional e emocional do divórcio, o sistema nuclear que modera, regula e potencia a mudança associada a esta transição é o sistema de vinculação.

Comitantemente, tendo como referência o adulto divorciado como pai e membro de uma família, e utilizando uma matriz sistémica, o divórcio é responsável por profundas alterações no sistema familiar, obrigando os seus subsistemas a proceder a reorganizações estruturais. A dissolução conjugal é um evento stressor do sistema familiar, sendo que a capacidade de absorção do seu impacto sistémico e a qualidade do funcionamento adaptativo dos filhos estão dependentes das características do 
sistema familiar durante o casamento (e.g., comunicação, laços afectivos, desempenho de papéis e flexibilidade relacional) e das propriedades da relação coparental mantida após o divórcio (Sbarra \& Emery, 2008). Por conseguinte, a cada um dos adultos é exigida a difícil tarefa de conciliar as necessidades desenvolvimentais dos seus filhos - o que sujeita o adulto a manter um contacto permanente e harmonioso e com baixos índices de conflito com o ex-cônjuge - e as suas próprias necessidades decorrentes do processo de adaptação ao divórcio (Adamsons \& Pasley, 2006). Contudo, os processos que explicam a adequada articulação entre a própria adaptação ao divórcio e as relações coparentais estão pouco explorados, sendo que, como defenderemos a seguir, a vinculação pode funcionar como um mapa conceptual válido na compreensão desta dinâmica familiar pós-divórcio.

Estas considerações prévias mostram a necessidade de explicitar com maior exactidão como a comunidade científica tem definido a coparentalidade. Segundo Mullett e Stolberg (1999), os pais separam-se do seu papel de parceiro romântico e íntimo, mas mantêm o seu estatuto e papel de companheiro coparental. Apesar de a coparentalidade ser um subsistema familiar transversal a todas as famílias, independentemente da sua configuração estrutural, a sua activação coordenada e eficiente após o divórcio torna-se fulcral, devido às suas características moderadoras do impacto da dissolução conjugal no desenvolvimento das crianças.

Erradamente, o conceito de coparentalidade é utilizado como sinónimo do grau de cooperação entre os pais na educação dos filhos, significando que os pais com boa cooperação exercitam a coparentalidade (Van Egeren \& Hawkins, 2004). No entanto, o construto da coparentalidade não induz uma tendência de qualidade das relações interparentais. Conceptualmente, a coparentalidade é definida como a relação diádica entre os pais na planificação e execução de um plano parental conjunto para os seus filhos (Feinberg, 2003), sendo que a coparentalidade positiva/cooperante é caracterizada pelo envolvimento recíproco e coeso da díade coparental na educação e tomada de decisões sobre a vida das crianças.

A inovação conceptual trazida pela coparentalidade é formular as relações interparentais como um subsistema autónomo e processual e estruturalmente diferenciável dos subsistemas conjugal e parental (Feinberg, 2003). Por consequência, a coparentalidade assume uma dimensão sistémica e triádica (pai-mãe-filho) (Caldera \& Lindsey, 2006).

Operacionalizando esta posição conceptual às famílias com pais divorciados, Maccoby, Depner e Mnookin (1990), no seu estudo de referência sobre os padrões relacionais entre díades parentais divorciadas, identificaram três variações da coparentalidade: a coparentalidade cooperativa, conflituosa ou descomprometida. A coparentalidade cooperativa é conseguida através de interacções de qualidade entre os pais, pautadas por uma comunicação regular sobre os filhos, caracterizada por níveis mínimos de conflito e ausência de estratégias de corrosão das interacções que cada pai mantém individualmente com os filhos. A cooperação coparental requer unidade e simetria nos planos parentais (Stright \& Neitzel, 2003), em que os pais funcionam como uma verdadeira equipa na condução da educação dos filhos, quer do ponto de vista instrumental, quer de um ponto de vista emocional e valorativo. Este subtipo de coparentalidade, considerado o mais saudável e mais promotor de oportunidades óptimas de desenvolvimento para as crianças, representa $25 \%$ das relações coparentais entre os pais divorciados (Maccoby et al., 1990). A coparentalidade conflituosa é, por sua vez, também pautada pela regularidade da comunicação entre os pais, no entanto esta comunicação contém elevados níveis de conflito, hostilidade, criticismo e competição que, devido a esta postura de adversários, provoca um curto-circuito nas tentativas de um trabalho coparental eficaz. Por último, na coparentalidade descomprometida, mais frequente em díades parentais divorciadas, existe um envolvimento de cada pai na vida da criança, mas este envolvimento não é conjunto, em que os pais praticam uma parentalidade paralela, pautada por uma comunicação mútua rudimentar em torno das questões educacionais dos filhos. A investigação tem demonstrado que esta comunicação rudimentar e práticas parentais paralelas amplificam a possibilidade de triangulações intergeracionais dentro da família. Macie (2002), por exemplo, identificou que dois terços das famílias com pais divorciados manifestavam inequívocas e poderosas alianças pai/mãe-filho, o que, devido à disrupção da relação coparental dos pais, estava também associado a elevados índices de ansiedade e de desadaptação nos filhos.

Tendencialmente, nos primeiros dois anos após o divórcio, a relação coparental é caracterizada pela existência de níveis moderados-elevados de conflito ou diminuído compromisso recíproco na educação dos filhos. Esta conflituosidade e descomprometimento parece traduzirse em práticas parentais paralelas e dessincronizadas, que contribuem para o enfraquecimento da percepção das crianças sobre a aliança parental e fomentam o conflito interparental e o desajustamento familiar (Nunes-Costa, Lamela, \& Figueiredo, 2009).

\section{A Vinculação na Vida Adulta}

A vinculação na vida adulta envolve múltiplos e complexos sistemas de regulação comportamental, emocional e das relações interpessoais (J. Feeney, 2008). Na vida adulta, a configuração e funções da vinculação tornam-se mais complexas, devido ao desenvolvimento de sistemas comportamentais de procura e prestação de cuidados, intimidade, comportamento sexual e de exploração (George \& Solomon, 1999). Nos últimos 25 anos, 
a maioria dos quadros conceptuais da vinculação na vida adulta assenta nos pressupostos teóricos de Bowlby e Ainsworth. Embora exista uma continuidade desenvolvimental da função da vinculação ao longo do ciclo de vida, a sua manifestação torna-se progressivamente mais integrada e cognitiva e emocionalmente mais complexa. Tradicionalmente, o estudo da vinculação na infância centra-se na dimensão comportamental, enquanto na vida adulta as dimensões representacionais, cognitivas, narrativas e auto-perceptivas ganham relevo na compreensão da vinculação (Hazan \& Shaver, 1994).

Weiss (1982) foi um dos pioneiros na apresentação das características da vinculação nos adultos. Define a vinculação na vida adulta como uma relação recíproca entre iguais, em que existe procura de proximidade, sendo que a separação, a ameaça de separação ou a diminuída responsividade contribui para o aumento da ansiedade devido ao sentimento de inacessibilidade da figura de vinculação. Tal como a criança, em momentos de distress e de ameaça, os adultos irão recorrer à figura de vinculação para regulação emocional e diminuição dos níveis de ansiedade. O autor considera que, em consonância com o funcionamento da infância, a vinculação na vida adulta é direccionada para uma figura que é tida como capaz de provisionar cuidados, sendo que o comportamento de vinculação permanece, mesmo nos momentos em que esta referência não está disponível. No entanto, a investigação tem demonstrado que, ao contrário do que acontece na infância, a vinculação na vida adulta pauta-se por relações recíprocas e horizontais, em que os parceiros prestam e procuram cuidados em momentos de percepção de perigo para o self (J. Feeney, 1999). Este balanceamento transaccional é essencial para uma visão segura do mundo que permite a exploração por parte de ambos os membros da díade. A qualidade, tonalidade e intencionalidade da procura e prestação de cuidados são os marcadores para a regulação, o investimento, a intimidade/distância, a qualidade da relação conjugal e a manutenção dos laços afectivos (B. Feeney, 2004; Lima, Vieira, \& Soares, 2006).

A singularidade da vinculação nos adultos é portanto a simetria no processo de procura e prestação de cuidados entre os membros da díade, uma vez que cada elemento desempenha simultaneamente o papel de figura de vinculação (que presta cuidados e contribui para a auto-regulação emocional e cognitiva do outro elemento) e o papel de figura vinculada (que recebe cuidados, protecção e segurança) (Hazan \& Zeifman, 1999; Weiss, 1982). Durante a construção e fortalecimento da relação íntima, os membros da díade aumentam gradualmente a procura e prestação de cuidados ao outro membro. Num primeiro momento, a prestação de cuidados envolve dimensões instrumentais e emocionais; no entanto, devido à segurança progressivamente conquistada na relação, os cuidados procurados pelo membro menos seguro tornam-se essencialmente emocionais, dado que se sen- te mais capaz de gerir por si próprio situações potencialmente desorganizantes. Esta reciprocidade na procura e prestação de cuidados promove um sentimento de segurança, uma vez que os elementos da díade confiam no outro como capaz de providenciar protecção, cuidado e conforto. Estes sentimentos de segurança e protecção derivados da relação de vinculação são essenciais porque permitem ao adulto explorar e desenvolver actividades e tarefas fora do contexto de base segura ( $\mathrm{Fa}-$ ria, 2008; J. Feeney, 2008; Hazan \& Zeifman, 1999).

Zeifman e Hazan (2008) sustentam que a função basilar da vinculação é a protecção e a segurança, em que o adulto, num momento de distress e fragilidade, procura conforto e segurança numa figura sensível e capaz de o auxiliar na sua reorganização psicológica e, num sentido mais etológico, competente para assegurar a sua sobrevivência. Para além desta função primária, as relações de vinculação na vida adulta garantem ainda um sentimento de pertença (também essencial para o funcionamento adaptativo do adulto), fomentam a construção conjunta de objectivos, metas desenvolvimentais e, finalmente, impulsionam e reforçam um sentido de competência (J. Feeney \& Noller, 1996; Fraley \& Shaver, 2000).

Um crescente número de estudos tem proposto que o estilo de vinculação é um importante preditor da qualidade e satisfação conjugais (J. Feeney, Noller, \& Callan, 1994), em que os padrões de comunicação e a manifestação de afectividade negativa funcionam como mediadores entre a segurança da vinculação e a satisfação na relação (Davila, Bradbury, \& Fincham, 1998; J. Feeney, 2008). Hazan e Shaver (1987) conceberam as relações românticas como um processo de vinculação, diferenciando três padrões de vinculação (inseguro-evitante, inseguro-ansioso e seguro), correspondentes aos padrões e à caracterização dos mesmos conceptualizada por Ainsworth (Ainsworth, Blehar, Waters, \& Wall, 1978). Estes padrões formam-se a partir das expectativas do sujeito acerca das respostas prováveis da figura de vinculação geradas nas interacções precoces, mais tarde representadas cognitivamente nos internal working models (Bowlby, 1973). São estes modelos que determinam o comportamento e as expectativas nos relacionamentos na vida adulta (Platts, Tyson, \& Mason, 2002).

Concebendo as relações íntimas como mais satisfatórias (Simpson, 1990), os adultos com um estilo seguro de vinculação estão disponíveis para a intimidade e proximidade emocional. Percepcionam-se como merecedores de cuidados, sinalizam adaptativamente as suas necessidades e consideram o parceiro como capaz de funcionar como base segura (Hazan \& Shaver, 1987). Os adultos seguros investem na relação íntima sem nela diluir a sua individualidade e autonomia. São também disponíveis, responsivos e prestadores de cuidados sensíveis e adequados. Identificando as pistas de distress do outro, respondem-lhes de forma célere e eficaz, satisfazendo as necessidades de cuidado do parceiro. Global- 
mente, indivíduos seguros sentem-se confortáveis com o comprometimento relacional e com a interdependência estabelecida com o companheiro (Mikulincer \& Shaver, 2007).

Por outro lado, adultos com vinculação insegura-ansiosa mostram elevado receio de abandono e dúvida sobre a disponibilidade afectiva do parceiro, o que os leva a ser hipervigilantes, desconfiados e ciumentos. Esta vigilância constante pode reforçar comportamentos negativos do parceiro. Todos os conflitos são percepcionados como ameaçadores para a relação e para o self e são amplificados e experienciados com tristeza e desespero. $\mathrm{O}$ afecto negativo do companheiro contribui para corroborar ruminações e pensamentos auto-depreciativos, causados pela raiva sentida perante a figura de vinculação (Davis et al., 2003). Tendem também a querer alcançar a intimidade e o comprometimento muito rapidamente, apresentando um acentuado investimento nas relações íntimas e desejo de fusão com o outro. A prestação de cuidados é caracterizada pela ansiedade, inconsistência, intrusividade e dúvidas sobre a qualidade dos cuidados providenciados (Simpson, 1990). A falta de confiança, aliada à necessidade de proximidade, faz com que se tornem excessivamente envolvidos, mas que, ao mesmo tempo, dificilmente sejam considerados uma base segura. $\mathrm{O}$ padrão de vinculação insegura-ansiosa leva ainda a comportamentos de dependência em relação ao parceiro, bem como a sentimentos de mal-estar e baixa auto-estima (Platts et al., 2002). Este padrão desencadeia comportamentos pouco assertivos, tanto por défice como por excesso de comportamentos de procura e prestação de cuidados.

Por sua vez, os adultos com vinculação insegura-evitante mostram menor investimento nas relações, percepcionam a intimidade como ameaçadora, minoram a necessidade de proximidade, valorizam a independência e auto-suficiência e receiam depender do outro e da relação (J. Feeney \& Noller, 1996; Hazan \& Shaver, 1987). Apresentam dificuldades em aumentar o grau de intimidade com o parceiro, rejeitando as tentativas de proximidade física e emocional, de comprometimento e interdependência. Os indivíduos com vinculação evitante geram as emoções de forma disfuncional e não procuram o apoio do outro em situações adversas. Ao nível da prestação de cuidados, mostram um padrão de indisponibilidade e fraca sensibilidade e responsividade. Este padrão é caracterizado por baixa empatia, pouca abertura e incapacidade para reconhecer com eficácia os sinais de mal-estar do outro, em que o cuidado é pouco efectivo e raramente contingente às pistas de procura de cuidado do parceiro. Por outro lado, a procura de cuidados no contexto da relação íntima reflecte pouca confiança nas capacidades do parceiro para proporcionar resposta as necessidades. Esta procura de cuidados é feita de forma nem efectiva nem assertiva e com reduzida demonstração da vulnerabilidade do próprio, o qual responde com elevados níveis de emocionalidade negativa (Mikulincer \& Shaver, 2007).

Por consequência, a satisfação com a relação conjugal tende consequentemente a ser proporcional à segurança da vinculação dos membros da díade. Kirkpatrick e Davis (1994), por exemplo, conferiram que as mulheres com vinculação insegura-ansiosa manifestavam menos satisfação e afecto e maiores níveis de conflito e ambivalência na relação do que as mulheres com um estilo de vinculação segura. Neste estudo, os homens com vinculação insegura-evitante apresentavam menor satisfação conjugal, menor intimidade, compromisso e interdependência, quando comparados com homens seguros. Em comparação com os inseguros-evitantes, os homens com um estilo de vinculação insegura-ansiosa mostravam maior paixão e maior compromisso na relação.

Nesta linha de pensamento, Simpson (1990) foi precursor na avaliação dos comportamentos de caregiving em casais a partir do método observacional. Mostrou que os homens evitantes apresentam comportamentos de prestação de cuidados negligentes, em momentos em que as suas companheiras se sentem vulneráveis na realização de determinadas tarefas induzidas em contexto laboratorial. Reforçou posteriormente estes dados, uma vez que encontrou que os homens evitantes respondem a sinais de distress das companheiras com desagrado e pouco suporte afectivo (Rholes, Simpson, \& Orina, 1999). A associação entre um padrão de vinculação pautado por elevada ansiedade e os comportamentos de caregiving prestados foi depois explorada posteriormente por Collins e Feeney (2000). Os homens identificados com um estilo de vinculação insegura-ansiosa demonstravam um défice no suporte às companheiras envolvidas em tarefas stressantes, sobretudo perante sinais pouco claros. O procurar e prestar cuidados é assim concebido por estes autores como um processo diádico e recíproco dependente do estilo de vinculação de ambos parceiros.

Deste modo, a teoria da vinculação traz, desta forma, uma maior densidade conceptual na compreensão da ruptura conjugal, tal como será proposto de seguida.

\section{Vinculação, Psicopatologia e Divórcio}

Existe uma elevada incidência e prevalência de sintomatologia psicopatológica na população divorciada. Todavia, estes índices não se distribuem aleatoriamente pelos adultos divorciados, operando um conjunto de factores de risco e de protecção que potenciam ou inibem o surgimento de respostas desajustadas crónicas. A nosso ver, tal como exposto anteriormente, o estilo de vinculação e qualidade da matriz relacional pré-divórcio são elementos-chave para a compreensão do mal-estar psicológico após divórcio. Como será visível, a história de vinculação dos adultos apresenta-se como preditor relevante na intensidade de duração da resposta psicopatológica após esta transição de vida. 
A associação entre a vinculação e o ajustamento psicológico tem sido evidenciada pela investigação (e.g., Mikulincer \& Shaver, 2007; Soares \& Dias, 2007). De acordo com a teoria da vinculação, as experiências relacionais ao longo do ciclo de vida contribuem para a saúde mental, uma vez que influenciam o desenvolvimento social e emocional e a qualidade, forma e intenção dos relacionamentos, nomeadamente na vida adulta. Por outras palavras, a forma como o adulto opera e compreende o mundo, adquire ou ignora a informação, interage no quadro das relações interpessoais e resolve os desafios desenvolvimentais, depende do seu estilo de vinculação. Numa matriz relacional, o indivíduo adquire competências e estratégias de regulação emocional, de acordo com o seu estilo de vinculação, o que pode promover ou dificultar a sua adaptação ao contexto (Soares \& Dias, 2007). Carlson e Sroufe (1995) teorizam que a ligação entre vinculação e psicopatologia está associada com a organização de expectativas, atitudes e emoções relacionadas com a vinculação que potenciam ou constrangem o funcionamento comportamental e sócio-emocional. Por consequência, a adaptação contextual, o estabelecimento de novas interacções interpessoais e a forma como o adulto explora o contexto é influenciada por processos de regulação emocional, cognitiva e comportamental, resultante das experiências de vinculação.

Considerando os pressupostos conceptuais da Psicopatologia do Desenvolvimento, a desadaptação psicológica surge quando existem padrões inflexíveis de comportamento perante tarefas desenvolvimentalmente desafiantes e geradoras de stress, em que, quanto maior for a rigidez e indiferenciação emocional, cognitiva e comportamental, maior a possibilidade da situação geradora de distress resultar em perturbação psicopatológica. Nesse sentido, os adultos com vinculação segura adquirem, ao longo do desenvolvimento, um sentido de competência interpessoal e desenvolvem padrões comportamentais, cognitivos e emocionais adaptativos e adequados, que lhes providenciam estratégias de coping capazes de responder às exigências contextuais e a situações de distress e que funcionam como factor protector contra as dificuldades e as perturbações psicopatológicas. A segurança da vinculação permite ao adulto gerir adaptativamente a afectividade negativa e recorrer a apoio emocional e instrumental quando necessário, funcionando como um factor de resiliência nas situações difíceis (Davis et al., 2003; Mikulincer \& Shaver, 2007).

Por consequência, ao invés dos seguros, os adultos inseguros, devido às suas experiências relacionais insatisfatórias, formam o self e avaliam o contexto desenvolvimental como instáveis e incertos, com pouca previsibilidade do suporte dos outros, para além de um reportório de estratégias de resolução problemas mais rígidas, menos flexíveis e em menor número. A vulnerabilidade à desadaptação psicológica dos adultos com um estilo de vinculação insegura associa-se a repetidas experiências de recepção de cuidados de figuras de vinculação inconsistentes ou indisponíveis e resulta em dificuldades, quer na auto-regulação emocional, quer na procura de cuidados (M. Dozier, Stovall-McCough, \& Albus, 2008). Mais concretamente, os adultos inseguros-ansiosos tendem a apresentar um padrão de regulação emocional pobre, o que faz perdurar estados de desajustamento que podem persistir após o desaparecimento efectivo da fonte de distress. As principais estratégias de coping são a preocupação crónica e as cognições negativas e catastróficas que podem determinar psicopatologia. Os adultos com vinculação insegura-evitante, devido a dificuldades de gestão emocional, tendem a bloquear e suprimir as emoções e cognições associadas a situações de distress e a manter crenças sobre a sua autosuficiência, invulnerabilidade e invencibilidade perante ameaças ao self (M. Dozier et al., 2008; Mikulincer \& Shaver, 2007).

Assim, a vinculação segura parece ser um importante factor protector para adaptação psicológica e para estados de organização da experiência adaptados face a situações de ameaça e distress, como é o caso do divórcio. Existe também uma elevada incidência e prevalência de perturbações psicológicas em adultos com estilos de vinculação insegura.

Desta forma, esta leitura desenvolvimental da resposta sintomalógica contribui para a construção da nossa primeira hipótese teórica.

Hipótese Teórica 1: A Adaptação ao Divórcio é Predita pelos Estilos de Vinculação dos Adultos ao Ex-cônjuge e Trata-se de um Processo de Perda da Figura da Vinculação, tal como Defendido por Bowlby (1980)

Considerando os pressupostos da vinculação na vida adulta e as suas implicações para o desenvolvimento de quadros de desajustamento psicológico face a exigentes acontecimentos de vida - tal como o divórcio - julgamos que os vários padrões e perfis de adaptação ao divórcio desenhados pela investigação empírica poderão ser conceptualizados à luz da teoria de vinculação. Esta é a hipótese que passamos a descrever.

Analisando cuidadosamente os pressupostos de Bowlby (1980) acerca da perda de uma figura de referência, conclui-se que a vinculação é um excelente roteiro para a compreensão dos comportamentos, emoções, percepções e significações das experiências associadas ao fim da relação marital. Davis et al. (2003) conceptualizam o divórcio como uma perda da figura de vinculação. A dissolução conjugal ceifa a trajectória prevista da relação de vinculação entre a díade, terminando projectos e metas de vida comuns. A dissolução conjugal, à semelhança da morte do cônjuge, desafia o processo de lidar com a perda. Aliás, hipotetizamos que a perda da figura de vinculação por divórcio pode ser mais destruturante para a organização da vinculação do que a perda por morte da figura de referência. Este nosso pressuposto encontra 
fundamentação na teoria de Bowlby, uma vez que, em situações de perda por morte, a pessoa sabe que a figura não providencia cuidados neste momento de stress por não existir fisicamente, no divórcio, a figura de vinculação é, por um lado, ela própria responsável pelo distress do adulto e, por outro lado, continua fisicamente presente, não providenciando deliberadamente cuidados ao ex-companheiro. Este quadro pode ser, a nosso ver, mais descompensador e desiquilibrador do sistema de vinculação.

Nesta linha, Bowlby (1980) propôs quatros períodos de um processo de luto (mourning) funcional e adaptativo que pode ser utilizado para a leitura das reacções comportamentais, cognitivas, emocionais e representacionais após a decisão de divórcio. Estas fases são numbing, procura e raiva, desorganização e desespero, e reorganização. A primeira fase, numbing (entorpecimento), é caracterizada por períodos de incredulidade sobre a perda da figura de vinculação, seguidos por intensas emoções e sintomas agudos de psicopatologia, como ansiedade e raiva. A segunda fase, caracterizada pela procura e raiva, envolve o início do registo da realidade da perda, acompanhado por um sentimento de inquietude e alvoroço, bem como de reacções de raiva com a finalidade de restabelecer o contacto com a figura de vinculação. A desorganização é a terceira fase do processo de luto, que inicia um período de confusão, agitação, incerteza e receio sobre a mudança. Neste período, o adulto aceita a perda após compreender que a figura de vinculação não voltará a estar disponibilidade, apesar do protesto constante em estabelecer contacto. Este protesto sem consequências pode resultar em manifestações de desespero, como depressão, tristeza, melancolia, confusão mental e sintomas psicopatológicos. A última fase do período de luto adaptativo é reorganização/integração, é caracterizada pela reestruturação gradual do self, das representações da figura de vinculação e por um processo de manutenção adaptativa dos laços. A adaptação à perda está associada a resignificações da inacessibilidade da figura de vinculação e a possibilidade da manutenção do laço com a figura de vinculação, após a alteração das suas funções e tonalidades emocionais.

Dessa feita, propomos que os adultos divorciados tendem a experienciar o processo de perda descrito por Bowlby no período posterior à dissolução conjugal. Assim, as respostas e reacções cognitivas, comportamentais e emocionais dos adultos divorciados serão muito similares às experienciadas pelos adultos viúvos, sendo que os estilos de vinculação irão mediar o grau de adaptação e reorganização psicossocial dos adultos ao divórcio, tal como acontece com os adultos viúvos. Alguns estudos corroboram esta nossa proposta de conceptualizar o divórcio como uma perda da figura de vinculação. Kitson (1982), por exemplo, observou que $86 \%$ dos adultos demonstravam comportamentos de vinculação para com o ex-cônjuge, associados à intensidade emocional partilhada entre a ex-díade durante a sua relação. Por seu lado,
Berman (1988) mostrou uma associação directa entre a frequência de imagens e pensamentos positivos do excônjuge com a ansiedade durante o processo de divórcio, em que quanto maior a segurança da vinculação, melhores estratégias de gestão emocional do distress resultante da dissolução conjugal (Cary, 2000). Segundo o estudo pioneiro de Bakermans-Kranenburg e van IJzendoorn (1997), a decisão de ruptura conjugal não é aleatória e mostrou-se directa e fortemente associada ao padrão de vinculação dos participantes no estudo. Esta investigação, baseada nos padrões de vinculação analisados através da Adult Attachment Interview (George, Kaplan, \& Main, 1985), concluiu, por um lado, que os adultos classificados com o estatuto de não-resolvidos quanto à perda por morte de uma pessoa de referência no passado são aqueles que indicam mais episódios de ruptura das relações íntimas, bem como reportam mais relacionamentos amorosos. Por outro lado, os adultos com um estilo de vinculação preocupado/ansioso tendem a retardar a decisão de dissolução relacional, não deixando o ex-parceiro iniciar o processo de divórcio (BakermansKranenburg \& van IJzendoorn, 1997).

Teoricamente, as respostas de cada adulto ao processo de divórcio estão significativamente associadas ao seu estilo de vinculação, sendo possível traçar um perfil preditivo da adaptação ao divórcio, de acordo com as características idiossincráticas a cada um dos estilos, descritas por nós no início desta secção. Resumidamente, do ponto de vista teórico, os adultos com um estilo de vinculação segura apresentam um vasto conjunto de recursos pessoais e contextuais que lhes permite proceder a uma transição adaptativa ao divórcio, enquanto os adultos com vinculação insegura tendem a percepcionar o divórcio como ameaçador que coloca em evidência os seus insuficientes recursos na gestão deste evento. Mais concretamente, os adultos seguros, devido às representações dos outros como responsivos e disponíveis, são capazes de assinalar eficazmente o distress trazido com a separação e sentem-se capazes de proceder às mudanças necessárias após o divórcio (Figueiredo \& Lamela, 2010; Vareschi \& Bursik, 2005). Ao contrário dos adultos seguros, os adultos com uma vinculação insegura-evitante tenderão a utilizar estratégias de coping baseadas na minimização da perda da figura de vinculação, apresentando altos índices de hostilidade e baixa sinalização do distress (Mikulincer \& Florian, 1998). Por outro lado, o divórcio para os adultos com vinculação insegura-ansiosa será lido como um contexto altamente hostil e ameaçador e, perante a indisponibilidade da figura de vinculação, terão maior dificuldade em reorganizar-se e integrar a experiência do divórcio (Davis et al., 2003).

Neste sentido, para os adultos com vinculação insegura, devido aos seus internal working models formados ao longo do ciclo de vida, o divórcio apresenta-se como um dos eventos mais destruturantes e os seus efeitos tendem a ser mais negativos e prolongados do que para os adultos seguros. Estes acentuados efeitos negativos são a 
resposta à separação permanente da figura de vinculação, o que confirma e valida as expectativas e receio de rejeição e indisponibilidade dos outros. De acordo com Mikulincer e Florian (1996, 1998), o divórcio pode causar a reactivação de separações não resolvidas de figuras de vinculação prévias, o que, associado a menores competências de coping e de regulação emocional, explica a maior probabilidade de problemas na adaptação ao divórcio e na integração da perda do ex-cônjuge e na reorganização dos laços de vinculação.

Por conseguinte, a nossa hipótese é suportada igualmente por vários estudos sobre os resultados adaptacionais dos adultos divorciados, uma vez que a teoria da vinculação consegue explicar as variações interindividuais a este acontecimento de vida. Em comparação com os casados, os adultos divorciados apresentam maior incidência de problemas psicopatológicos, isolamento, raiva, hostilidade, infelicidade e sentimentos de incompetências e rejeição (Amato, 2001; Gähler, 2006). Alguns dados sugerem que os sintomas de distress psicológico podem ser esperados nos períodos imediatos à dissolução conjugal, uma vez que Booth e Amato (1991) mostram que o distress psicológico é significativamente mais elevado nos momentos imediatos ao divórcio e diminui gradualmente de intensidade nos anos seguintes. Este padrão confirmado em vários estudos vai claramente de encontro às fases do processo de luto da figura vinculação descrito por Bowlby (1980). A nosso ver, a resposta psicopatológica acentuada pode ser resultado do colapso das estratégias de regulação do distress causado pelo divórcio. Mikulincer e Shaver (2007), baseados em Bowlby, dizem que o sistema de vinculação na vida adulta pode ser activado devido a três fontes de distress: ameaça real ou percebida à pessoa ou ao self, ameaça real ou percebida à relação de proximidade com a figura de vinculação ou, finalmente, outras situações desafiadoras que impulsionam a pessoa a recorrer ao cuidados da figura de vinculação. De facto, na nossa perspectiva, o divórcio pode activar ao mesmo tempo estas três fontes de distress, uma vez que a dissolução conjugal contribui para visões distorcidas do self, da competência e com sentimentos de culpa, coloca efectivamente a figura de vinculação indisponível num momento, por ele próprio, altamente desafiador e ameaçador devido, por exemplo, à necessidade de mudar de casa, encontrar emprego, refazer redes sociais. Por consequência, devido à conjugação sincrónica destas fontes de stress, as estratégias de regulação emocional podem entrar em colapso por saturação e ruptura no período imediatamente a seguir ao divórcio, em especial em adultos com estilos de vinculação insegura.

Inerentemente, a visão da adaptação ao divórcio como um processo de vinculação remete automaticamente para análise das relações coparentais pós-divórcio à luz dos processos de desvinculação ao ex-cônjuge, como descrevemos de seguida.

\section{Vinculação, Divórcio e Coparentalidade}

Hipótese Teórica 2: A Reorganização Familiar Após o Divórcio pode ser Afectada Negativamente pelos Processos de Desvinculação ao Ex-companheiro e pelos Estilos de Vinculação dos Pais

$\mathrm{O}$ divórcio não anuncia o fim da família. Embora os pais tenham dissolvido o seu estatuto de parceiro íntimo, continuam a manter o seu papel de díade parental e coparental. Nas últimas décadas um consistente corpo de investigação tem demonstrado ligações moderadoras entre a qualidade conjugal e o funcionamento dos filhos (Gadeyne, Ghesquière, \& Onghena, 2004).

No entanto, estas ligações moderadoras têm sido parcialmente rejeitadas, uma vez que pesquisas mais recentes têm comprovado que os percursos desenvolvimentais das crianças podem ser melhor explicados pela qualidade da coparentalidade e o seu impacto nas relações familiares, do que pela qualidade conjugal e parental per se. Por exemplo, Belsky, Crnic e Gable (1995) sustentam que a coparentalidade pode ser o factor mediador entre a qualidade das interacções da díade conjugal e os percursos desenvolvimentais ajustados dos filhos, uma vez que a coparentalidade pontua como o funcionamento conjugal influencia a parentalidade e, por consequência, a adaptação da fratia. Este valor mediador da coparentalidade tem poder preditivo na compreensão de como o conflito na díade conjugal é ampliado na relação coparental, diminuindo a eficácia da relação parental.

A perda da relação conjugal por si só, associada ao contacto e envolvimento contínuos com o ex-cônjuge no exercício da coparentalidade, tem sido vista, do ponto de vista da teoria da vinculação, como o acontecimento mais stressante da experiência de divórcio (B. Feeney \& Monin, 2008). O distress sentido na transição do divórcio deve-se a sentimentos de vinculação pelo ex-companheiro que podem causar problemas emocionais e de ajustamento após a separação. Tendo em consideração esta premissa, estudos demonstram que a manutenção de contactos com o ex-cônjuge - e figura de vinculação está associada com piores índices de ajustamento ao divórcio (e.g., Duran-Aydintug, 1995). No entanto, outras investigações empíricas concluem que o adulto exerce o seu papel parental com maior eficácia quando os pais mantêm contactos após a dissolução conjugal (e.g., B. Dozier, Sollie, Stack, \& Smith, 1993). O equilíbrio adaptativo entre o papel coparental e os projectos pessoais após o divórcio é dependente dos estilos de vinculação e dos internal working models, como veremos de seguida.

Não será extremo afirmar que o papel da coparentalidade é central nas relações familiares após a dissolução conjugal (Hetherington \& Kelly, 2002). Assim, o que difere as famílias binucleares das famílias uninucleares é o esforço e a dificuldade na criação e manutenção de uma aliança cooperante entre os pais, mais dificultada 
nos pais divorciados. Estas dificuldades acrescidas resultam, por um lado, dos conflitos pré e pueri divórcio, uma vez que os pais tendem a estar vinculados a padrões de interacção coercivos que ditaram a dissolução do casamento e, por outro, da necessidade de reorganização familiar e estabelecimento de novas fronteiras e papéis relacionais (Madden-Derdich, Leonard, \& Christopher, 1999).

A reorganização familiar pode ser afectada negativamente pelos processos de desvinculação - ou reorganização da vinculação, segundo a terminologia de Bowlby (1980) - ao ex-companheiro. Baseados em alguns dados empíricos, levantamos a hipótese que pessoas que apresentam um padrão vinculação insegura-ansiosa, a esfera coparental tende a ser utilizada para expressar o descontentamento e irritabilidade pela indisponibilidade da figura de vinculação em prestar cuidados neste período agudo de stress. De facto, a qualidade da relação coparental está associada aos níveis de desvinculação ao ex-parceiro e à diminuição da manifestação de comportamentos de vinculação. Masheter (1997) afirma que a vinculação emocional ao ex-parceiro pode ser manifestada por preocupação ou hostilidade e rejeição perante o ex-companheiro. Sendo que, a maioria dos ex-casais, apenas mantêm contacto devido às questões coparentais, os comportamentos de vinculação tendem a manifestarse com mais intensidade nesta área relacional, o que mina as possibilidades de uma coparentalidade cooperante.

Hetherington e Stanley-Hagen (1999) observaram que apenas um quarto das díades coparentais têm uma interacção marcada pelo conflito dois anos após o divórcio, o que denota que há um aumento das interacções positivas e qualidade da coparentalidade com o avançar dos anos do divórcio. Esta melhoria da coparentalidade poderá estar associada ao processo de desvinculação ao ex-parceiro, uma vez que, tendo por base o modelo processual de perda Bowlby (1980), julgamos que as respostas iniciais à perda do parceiro (e.g., descrença, ansiedade, depressão, raiva e desespero) poderão dificultar a manutenção de uma relação coparental positiva com ex-parceiro, não facilitando o diferenciar e distinguir da relação conjugal passada da relação coparental do presente. Dessa forma, e de acordo com a nossa proposta teórica, uma coparentalidade positiva poderá ser apenas alcançada no período de reorganização da vinculação, após a resignificação da perda da figura da vinculação. E esta reorganização mais ou menos adaptada está certamente associada ao estilo de vinculação dos elementos envolvidos no processo. Alguns dados sustentam esta formulação. Figueiredo e Lamela (2010), por exemplo, encontram uma forte associação entre a vinculação insegura e o desajustamento ao divórcio e ausência de coparentalidade positiva em adultos recém-divorciados. Mais concretamente, evidenciaram que a vinculação insegura-evitante dos adultos prediz, nos dois primeiros meses após o divórcio, relações coparentais conflituosas ou descomprometidas. Estes dados confirmam as con- clusões do estudo de Madden-Derdich e Arditti (1999) que observaram que, nos períodos iniciais ao divórcio, interacções coparentais positivas e cooperantes predizem a activação de comportamentos de vinculação, em que quanto maior forem as interacções coparentais, mais comportamentos de preocupação de vinculação e pior ajustamento ao divórcio das mães. Dessa forma, embora as relações coparentais suportivas possam contribuir para a adaptação dos filhos, elas podem dificultar o processo de luto e desvinculação da figura de vinculação e não promover a adaptação ao divórcio. Julgamos este dado de maior relevância, que carece de investigação sistemática.

\section{Discussão}

A finalidade do presente artigo era apresentar a vinculação como o processo explicativo da adaptação dos adultos ao divórcio, bem como das relações coparentais após a dissolução conjugal. Sustentando-nos na reduzida investigação empírica publicada, operacionalizámos a interligação destes construtos através de duas hipóteses teóricas.

Mais concretamente, na hipótese teórica 1, defendemos que o divórcio desafia ao adulto a lidar com a perda da figura da vinculação. Argumentámos que a conceptualização do divórcio como um evento que activa o sistema de vinculação permite compreender, por um lado, as respostas sintomatológicas dos adultos e, por outro lado, explicar as interacções comportamentais desajustadas e negativas entre os ex-cônjuges no período imediato à efectivação da separação.

Baseados no modelo de perda de Bowlby (1980), considerámos que a adaptação ao divórcio pode ser predita pelo processo de adaptação à perda da figura de vinculação, o que pode explicar os níveis de desadaptação global normativos durante o primeiro/segundo ano após a dissolução conjugal. Nesta linha, defendemos igualmente que o conflito, que acompanha a maioria dos casos de divórcio, pode ser lido como um conjunto de comportamentos (e.g., protesto, hostilidade e raiva) que visam a manutenção da relação de vinculação. Assim, quando a separação é processada como temporária, ao contrário de que outros modelos de adaptação ao divórcio preconizam (e.g., Wallerstein, Lewis, \& Blakeslee, 2000; Wang \& Amato, 2000), o conflito durante os meses seguintes ao divórcio pode ser funcional, uma vez que tem como finalidade sinalizar o distress, requerer a atenção da figura de vinculação e assegurar que uma separação futura não ocorra (Toews, McKenry, \& Catlett, 2003; Vareschi \& Bursik, 2005).

Nesta linha, propusemos que a resposta psicopatológica e desajustamento psicossocial comuns a uma grande maioria dos adultos que experienciam este evento podem ser considerados como resultados deste processo de reorganização da vinculação, sendo que a qualidade e complexidade desta reorganização se encontra 
dependente dos estilos de vinculação dos adultos. A importância dos estilos de vinculação na compreensão das dinâmicas de adaptação ao divórcio prende-se com o valor compósito deste construto, uma vez que possibilitam, tal como foi descrito por nós, a predição das diferenças interindividuais ao nível da qualidade do relacionamento interpessoal, da regulação emocional, das estratégias de prestação e procura de cuidados, das competências de coping e do processamento e acção de/ sobre as ameaças.

Sendo assim, esta hipótese contribui, implicitamente, para despatologização das respostas psicopatológicas e condutas conflituosas após o divórcio. Ao invés de tais comportamentos serem lidos como desadequados e incapacitantes, eles assumem um carácter adaptativo e essencial ao processo de reorganização da experiência e no planeamento de novas rotas desenvolvimentais. Dessa forma, nos primeiros períodos após a dissolução do casamento, estas respostas lidas como desadaptativas são conceptualizadas, à luz da teoria da vinculação, como indicadores adaptativos da gestão e integração emocional, cognitiva e comportamental do divórcio e das mudanças que este evento implica.

Retomando novamente a perspectiva sobre a perda de Bowlby, este contributo teórico fornece ideias relevantes para o estudo do divórcio. Face à perda da figura de vinculação, Bowlby defende que a última fase do processo de adaptação à perda é a reorganização da vinculação. Tal como referem Shaver e Fraley (2008), Bowlby utiliza o conceito de "reorganização" em vez de "desvinculação", o que implica que o autor considera que exista uma continuação, aos níveis representacional e emocional, dos laços entre a figura vinculada e a figura de vinculação, entendendo-se implicitamente a quase impossibilidade de diluir esta relação de vinculação.

No divórcio, tal proposição significa que níveis elevados de bem-estar e de adaptação ao divórcio não estão dependentes de uma desvinculação do ex-cônjuge, mas sim da reorganização da relação interpessoal com o excônjuge. B. Feeney e Monin (2008) assumem um pressuposto similar, acrescentando que este processo de reorganização e de redefinição é alcançado através da transição de um laço de vinculação entre os ex-cônjuges para um laço de afiliação, que depende de um sistema comportamental separado (B. Feeney \& Monin, 2008).

Ao longo do artigo, argumentámos que este processo de reorganização da vinculação face ao ex-cônjuge é particularmente relevante entre os adultos que partilham a parentalidade. Na hipótese teórica 2, sustentámos que o processo de reorganização face à perda da figura de vinculação, mediada e moderada pelos estilos de vinculação dos pais, interfere e prediz a qualidade das relações coparentais após o divórcio. Por conseguinte, apresentámos a hipótese que os comportamentos de raiva e hostilidade perante a não resposta da figura de vinculação ao distress do adulto, característicos no período inicial da reorganização da vinculação, são manifestados na esfera coparental, uma vez que tende ser o único contexto relacional mantido pelos ex-cônjuges no exercício conjunto das responsabilidades parentais.

De acordo com a nossa segunda hipótese, a prática adaptativa da coparentalidade pode estar condicionada pelo processo de adaptação ao divórcio de cada um dos pais, sendo que a capacidade de conciliar e distinguir as necessidades pessoais de cuidado, idiossincráticas à relação conjugal terminada, e as responsabilidades coparentais é regulada pelos estilos de vinculação de cada um dos membros da díade coparental. Baseados na rara investigação empírica, sustentámos que os adultos seguros, devido ao seu repertório comportamental, emocional e cognitivo, mostram-se mais capazes, por um lado, de separar as suas próprias necessidades das exigências coparentais e, por outro lado, de estabelecer um novo código relacional com o ex-cônjuge, potenciando a relação de vinculação passada numa relação coparental desenvolvimentalmente adequada e responsiva às necessidades dos filhos. Nesta linha de pensamento, os pais com estilos de vinculação insegura, apresentam-se menos capazes de diferenciar e gerir a relação de vinculação da relação coparental, o que se reflecte na maior probabilidade de conflituosidade crónica e litigância judicial na regulação da aliança coparental.

Durante o processo de divórcio, os laços de vinculação são rompidos, precipitando a activação do sistema de vinculação com a finalidade de preservar o laço emocional/relacional. De facto, as reacções e processos de adaptação psicossocial à dissolução conjugal podem ser integrados teoricamente à luz da teoria da vinculação, uma vez que a segurança da vinculação está estritamente associada à adaptação positiva dos adultos divorciados. Neste sentido, a partir da teoria da vinculação, o divórcio pode ser formulado como um evento stressante que activa o sistema de vinculação na vida adulta, tal como a Situação Estranha para a criança. A dissolução conjugal certamente activa as estratégias de vinculação e as diferenças individuais podem ser melhor compreendidas quando é tomada em consideração o self e as relações actuais e passadas com os outros. Reforçando este pressuposto, e analisando as conclusões de Berman (1988) e Kitson (1982), a vinculação é um dos factores mais significativos do processo de divórcio, uma vez que estes estudos evidenciaram que a vinculação explica com mais exactidão as experiências de adaptação ao divórcio do que outras variáveis como a idade, o género, o distress global ou o nível sócio-económico.

No entanto, estes resultados devem ser reforçados com novas pesquisas empíricas. A maioria dos estudos publicados sobre as implicações da vinculação na adaptação à dissolução de relações íntimas circunscreve-se a relações de namoro em jovens adultos. No entanto, há alguns dados que indicam que o impacto da dissolução do casamento no funcionamento psicológico é mais severo do que é assistido no fim de um namoro (Hill, Rubin, \& Peplau, 1976), pelo que é necessário em investigações 
futuras proceder a estudos comparativos entre os vários tipos de dissolução das relações íntimas e utilizando métodos de avaliação da vinculação validados.

A qualidade das interacções dentro sistema familiar está dependente do funcionamento e o bem-estar psicológicos de cada um dos seus membros. A partir desta perspectiva dos sistemas familiares, parece linear que a adaptação dos membros do ex-casal ao divórcio irá influenciar as relações coparentais entre si e, se a adaptação à dissolução conjugal tem sido associada pela investigação psicológica aos estilos de vinculação, não será errado concluir que a aliança coparental entre pais divorciados possa ser explicada, em grande medida, pelos padrões de vinculação dos adultos. Alguns autores confirmam este pressuposto; no entanto, não existem indicações empíricas suficientes de como as estratégias de vinculação dos pais influenciam a relação coparental que mantêm com o seu ex-companheiro. Enquanto Ahrons e Rodgers (1981) consideram que os estudos sobre a vinculação no divórcio se debruçam nos aspectos negativos da vinculação ao ex-cônjuge e afirmam que algumas dimensões da vinculação para além da decisão legal de divórcio podem ter consequências positivas para a família na relação coparental e na formação de uma relação mais construtiva entre a ex-díade, estudos mais recentes (e.g., Figueiredo \& Lamela, 2010; MaddenDerdich et al., 1999) levantam reticências quanto a esta posição teórica de Ahrons e Rodgers (1981), devido à dificuldade em estabelecer um parecer robusto entre o impacto da vinculação ao ex-cônjuge no ajustamento psicológico adulto divorciado. Apesar de a curto-prazo serem possíveis efeitos positivos da não ruptura do laço nas relações coparentais (e.g. Ahrons \& Rodgers, 1981; Madden-Derdich et al., 1999), não estão ainda descritos os efeitos a médio e longo prazo no funcionamento, nas fronteiras, limites, regras e processos de interacção emocional dentro do sistema familiar. Por consequência, são prioritários projectos de investigação que tenham como finalidade contribuir para a clarificação das relações entre adaptação ao divórcio, vinculação e coparentalidade. Para esta análise são necessários desenhos metodológicos robustos que permitam compreender a variabilidade intraindividual nos processos de vinculação e coparentalidade ao longo do tempo de dissolução conjugal.

\section{Referências}

Adamsons, K., \& Pasley, K. (2006). Coparenting following divorce and relationship dissolution. In M. Fine \& J. Harvey (Eds.), Handbook of divorce and relationship dissolution (pp. 241-262). Mahwah, NJ: Lawrence Erlbaum.

Ahrons, C., \& Rodgers, R. (1981). Divorced families. New York: Norton.

Ainsworth, M., Blehar, M., Waters, E., \& Wall, S. (1978). Patterns of attachment. Hillsdale, NJ: Lawrence Erlbaum.

Amato, P. (2001). Children of divorce in the 1990s: An update of the Amato and Keith (1991) meta-analysis. Journal of Family Psychology, 15, 355-370.
Bakermans-Kranenburg, M., \& van IJzendoorn, M. (1997) Adult attachment and the break-up of romantic relationships. Journal of Divorce \& Remarriage, 27, 121-141.

Belsky, J., Crnic, K., \& Gable, S. (1995). Determinants of coparenting in families with toddler boys: Spousal differences and daily hassles. Child Development, 66, 629-642.

Berman, W. (1988). The role of attachment in the post-divorce experience. Journal of Personality and Social Psychology, 53, 496-503.

Booth, A., \& Amato, P. (1991). Divorce and psychological stress. Journal of Health and Social Behavior, 32, 396-407.

Bowlby, J. (1973). Attachment and loss: Separation, anxiety, and anger. New York: Basic Books.

Bowlby, J. (1980). Attachment and loss: Vol. 3. Loss: Sadness and depression. New York: Basic Books.

Braver, S., Shapiro, J., \& Goodman, M. (2006). The consequences of divorce for parents. In M. Fine \& J. Harvey (Eds.), Handbook of divorce and relationship dissolution (pp. 313338). Mahwah, NJ: Lawrence Erlbaum.

Caldera, Y. M., \& Lindsey, E. W. (2006). Coparenting, motherinfant interaction, and infant-parent attachment relationships in two-parent families. Journal of Family Psychology, 20, 275-283.

Carlson, E., \& Sroufe, A. (1995). Contribution of attachment theory to Developmental Psychopathology. In D. Cicchetti \& D. Cohen (Eds.), Development and Psychopathology: Vol. 1: Theory and method (pp. 581-617). New York: Wiley.

Cary, H. (2000). Attachment status and post-divorce adjustment in women. Unpublished doctoral dissertation, California School of Professional Psychology, Alameda, CA.

Cavanaugh, J., \& Blanchard-Fields, F. (2009). Adult development and aging. Belmont, CA: Wadsworth.

Collins, N., \& Feeney, B. (2000). A safe haven: Support-seeking and caregiving processes in intimate relationships. Journal of Personality and Social Psychology, 78, 1053-1073.

Davila, J., Bradbury, T., \& Fincham, F. (1998). Negative affectivity as a mediator of the association between adult attachment and marital satisfaction. Personal Relationships, 5, 467-484.

Davis, D., Shaver, P., \& Vernon, M. (2003). Physical, emotional, and behavioral reactions to breaking up: The roles of gender, age, emotional involvement, and attachment style. Personality and Social Psychology Bulletin, 29, 871-884.

Dozier, B., Sollie, D., Stack, J., \& Smith, A. (1993). The effects of postdivorce attachment on coparenting relationships. Journal of Divorce and Remarriage, 19, 109-123.

Dozier, M., Stovall-McCough, C., \& Albus, K. (2008). Attachment and psychopathology in adulthood. In J. Cassidy \& P. R. Shaver (Eds.), Handbook of attachment: Theory, research, and clinical applications ( $2^{\text {nd }}$ ed., pp. 718-744). New York: Guilford Press.

Duran-Aydintug, C. (1995). Former spouse interaction: Normative guidelines and actual behavior. Journal of Divorce \& Remarriage, 22, 147-161.

Faria, C. (2008). Vinculação e desenvolvimento epistemológico em jovens adultos. Tese de Doutorado não-publicada, Universidade do Minho, Braga, Portugal.

Feeney, B. (2004). A secure base: Responsive support of goal strivings and exploration in adult intimate relationships. Journal of Personality and Social Psychology, 87, 631-648.

Feeney, B. C., \& Monin, J. K. (2008). An attachment-theoretical perspective on divorce. In J. Cassidy \& P. R. Shaver (Eds.), Handbook of attachment: Theory, research, and clinical applications ( $2^{\text {nd }}$ ed., pp. 934-957). New York: Guilford. 
Feeney, J. (1999). Issues of closeness and distance in dating relationships: Effects of sex and attachment style. Journal of Social and Personal Relationships, 16, 571-590.

Feeney, J. (2008). Adult romantic attachment and couple relationships. In J. Cassidy \& P. R. Shaver (Eds.), Handbook of attachment: Theory, research, and clinical applications ( $2^{\text {nd }}$ ed., pp. 456-481). New York: Guilford.

Feeney, J., \& Noller, P. (1996). Adult attachment. Thousand Oaks, CA: Sage.

Feeney, J., Noller, P., \& Callan, V. (1994). Attachment style, communication and satisfaction in the early years of marriage. Advances in Personal Relationships, 5, 269-308.

Feinberg, M. (2003). The internal structure and ecological context of coparenting: A framework for research and intervention. Parenting: Science and Practice, 3, 95-131.

Figueiredo, B., \& Lamela, D. (2010). Marital intimate relationship before divorce: Psychological adjustment and co-parenting relationship after marital dissolution. Submetido para publicação.

Fraley, R., \& Shaver, P. (2000). Adult romantic attachment: Theoretical developments, emerging controversies, and unanswered questions. Review of General Psychology, 4, $132-154$

Gadeyne, E., Ghesquière, P., \& Onghena, P. (2004). Longitudinal relations between parenting and child adjustment in young children. Journal of Clinical Child \& Adolescent Psychology, 33, 347-358.

Gähler, M. (2006). "To divorce is to die a bit...": A longitudinal study of marital disruption among Swedish women and men. The Family Journal, 14, 372-382.

George, C., Kaplan, M., \& Main, M. (1985). Adult attachment interview. Unpublished manuscript, Universidade da Califórnia, Berkeley, CA.

George, C., \& Solomon, J. (1999). Attachment and caregiving: The caregiving behavioural system. In J. Cassidy \& P. R. Shaver (Eds.), Handbook of attachment: Theory, research and clinical applications (pp. 649-670). New York: Guilford Press

Greene, S., Anderson, E., Hetherington, M., Forgatch, M., \& DeGarmo, D. (2006). Risk and resilience after divorce. In F. Walsh (Ed.), Normal family processes $\left(3^{\text {rd }}\right.$ ed., pp. 96120). New York: Guilford.

Hazan, C., \& Shaver, P. (1987). Romantic love conceptualized as an attachment process. Journal of Personality and Social Psychology, 59, 511-524.

Hazan, C., \& Shaver, P. (1994). Attachment as an organizational framework for research on close relationships. Psychological Inquiry, 5, 1-22.

Hazan, C., \& Zeifman, D. (1999). Pair bonds as attachments: Evaluating the evidence. In J. Cassidy \& P. R. Shaver (Eds.), Handbook of attachment: Theory, research, and clinical applications (pp. 336-354). New York: The Guilford Press.

Hetherington, M. (2006). The influence of conflict, marital problem solving and parenting on children's adjustment in nondivorced, divorced and remarried families. In A. ClarkeStewart \& J. Dunn (Eds.), Families count: Effects on child and adolescent development (pp. 203-237). New York: Cambridge University Press.

Hetherington, M., \& Kelly, J. (2002). For better or for worse: Divorce reconsidered. New York: Norton.

Hetherington, M., \& Stanley-Hagen, M. (1999). The adjustment of children with divorced parents: A risk and resiliency perspective. Journal of Child Psychology, 40, 129-140.
Hill, C., Rubin, Z., \& Peplau, L. (1976). Breakups before marriage: The end of 103 affairs. Journal of Social Issues, 32, 147-168.

Holmes, T., \& Rahe, R. (1967). The social readjustment rating scale. Journal of Psychosomatic Research, 11, 213-218.

Kalmijn, M., \& Poortman, A. (2006). His or her divorce? The gendered nature of divorce and its determinants. European Sociological Review, 22, 201-214.

King, L., \& Raspin, C. (2004). Lost and found possible selves, subjective well-being, and ego development in divorced women. Journal of Personality, 72, 603-632.

Kirkpatrick, L., \& Davis, K. (1994). Attachment style, gender and relationship stability: A longitudinal analysis. Journal of Personality and Social Psychology, 66, 502-512.

Kitson, G. (1982). Attachment to the spouse in divorce: A scale and its application. Journal of Marriage and Family, 44, 379-393.

Lamela, D. (2009). Desenvolvimento após o divórcio como estratégia de crescimento humano. Revista Brasileira de Crescimento e Desenvolvimento Humano, 19, 114-121.

Lima, V., Vieira, F., \& Soares, I. (2006). Vinculação em casais: Avaliação da representação da intimidade e da interacção conjugal. Psicologia, 20(1), 51-63.

Maccoby, E., Depner, C., \& Mnookin, R. (1990). Coparenting in the second year after divorce. Journal of Marriage and Family, 52, 141-155.

Macie, K. M. (2002). Influence of co-parenting and marital status on young adult adjustment. Unpublished doctoral dissertation, Virginia Commonwealth University, Richmond, VA.

Madden-Derdich, D., \& Arditti, J. (1999). The ties that bind: Attachment between former spouses. Family Relations, 48 , 243-249.

Madden-Derdich, D., Leonard, S., \& Christopher, F. (1999). Boundary ambiguity and coparental conflict after divorce: An empirical test of a family systems model of the divorce process. Journal of Marriage and Family, 61, 588-598.

Masheter, C. (1997). Healthy and unhealthy friendship and hostility between ex-spouse. Journal of Marriage and Family, 59, 463-475

Mikulincer, M., \& Florian, V. (1996). Coping and adaptation to trauma and loss. In M. Zeidner \& N. S. Endler (Eds.), Handbook of coping: Theory, research, applications (pp. 554-572). New York: John Wiley \& Sons.

Mikulincer, M., \& Florian, V. (1998). The relationship between adult attachment styles and emotional and cognitive reactions to stressful events. In J. A. Simpson \& W. S. Rholes (Eds.), Attachment theory and close relationships (pp. 143-165). New York: Guilford.

Mikulincer, M., \& Shaver, P. (2007). Attachment in adulthood: Structure, dynamics, and Change. New York: The Guildford Press.

Mullett, E., \& Stolberg, A. (1999). The development of the Co-parenting Behaviors Questionnaire: An instrument for children of divorce. Journal of Divorce \& Remarriage, 31, 115-137.

Nunes-Costa, R., Lamela, D., \& Figueiredo, B. (2009). Adaptação psicossocial e saúde física em crianças de pais separados. Jornal de Pediatria, 85, 385-396.

Platts, H., Tyson, M., \& Mason, O. (2002). Adult attachment style and core beliefs: Are they linked. Clinical Psychology and Psychotherapy, 9, 332-348. 
Rholes, W., Simpson, J., \& Orina, M. (1999). Attachment and anger in an anxiety-provoking situation. Journal of Personality and Social Psychology, 76, 940-957.

Sbarra, D., \& Emery, E. (2008). Deeper into divorce: Using actor-partner analyses to explore systemic differences in coparenting conflict following custody dispute resolution. Journal of Family Psychology, 22, 144-152.

Shaver, P., \& Fraley, R. C. (2008). Attachment, loss, and grief: Bowlby's views and current controversies. In J. Cassidy \& P. R. Shaver (Eds.), Handbook of attachment: Theory, research, and clinical applications $\left(2^{\text {nd }}\right.$ ed., pp. 48-77). New York: Guilford Press.

Simpson, J. A. (1990). The influence of attachment styles on romantic relationships. Journal of Personality and Social Psychology, 59, 971-980.

Soares, I., \& Dias, P. (2007). Apego y psicopatología en jóvenes y adultos: contribuciones recientes de la investigación. International Journal of Clinical and Health Psychology, 7, 177-195.

Stright, A. D., \& Neitzel, C. (2003). Beyond parenting: Coparenting and children's classroom adjustment. International Journal of Behavioral Development, 27, 31-40.

Toews, M., McKenry, P., \& Catlett, B. (2003). Male-initiated partner abuse during marital separation prior to divorce. Violence and Victims, 18, 387-402.

Van Egeren, L., \& Hawkins, D. (2004). Coming to terms with coparenting: Implications of definition and measurement. Journal of Adult Development, 11, 165-178.

Vareschi, C., \& Bursik, K. (2005). Attachment style differences in the parental interactions and adaptation patterns of divorcing parents. Journal of Divorce and Remarriage, 42, 15-32.

Wallerstein, J., Lewis, J., \& Blakeslee, S. (2000). The unexpected legacy of divorce. New York: Hyperion.

Wang, H., \& Amato, P. (2000). Predictors of divorce adjustment: Stressors, resources, and definitions. Journal of Marriage and Family, 62, 655-668.

Weiss, R. S. (1982). Attachment in adult life. In C. M. Parkes \& J. Stevenson-Hinde (Eds.), The place of attachment in human behaviour (pp. 171-194). New York: Basic Books.

Zeifman, D., \& Hazan, C. (2008). Pair bonds as attachments: Reevaluating the evidence. In J. Cassidy \& P. R. Shaver (Eds.), Handbook of Attachment: Theory, research, and clinical applications ( $2^{\text {nd }}$ ed., pp. 436-455). New York: The Guilford Press. 